\title{
Investigation of events in the EEG signal correlated with changes in both oxygen and glucose in the brain
}

\author{
Sabrina Valente", John Ringwood ${ }^{*}$, Violeta Mangourova*, John Lowry ${ }^{* *}$ \\ *Department of Electronic Engineering \\ ** Department of Chemistry \\ National University of Ireland, Maynooth \\ National University of Ireland, Maynooth \\ email : valentesabrina87@gmail.com \\ email: john.lowry@nuim.ie
}

\begin{abstract}
Since the brain has no constant energy reserves, a continuous supply of energy substrates is central to all processes that maintain the functionality of the neuronal cells. EEG has been found to be tightly related to variations in the concentration of the energy substrates such as oxygen and glucose. Prediction of neural activation is particularly useful as it could contribute significantly in the prevention, stabilization, or treatment of diseases such as Alzheimer's disease, migraine headache, and ischemic stroke, in which signaling between neurons and brain vessels is threatened because of dysfunctions that affect the neuronal, astroglial, and/or vascular components of the neurovascular unit. This work deals with investigation of events in the EEG signal correlated with changes in both oxygen and glucose signals in the brain. The topic is to implement a model that through measures of oxygen and glucose in the brain of rats allow to achieve a good estimation of the neural signals, which reflecting the simultaneous metabolic changes, during spontaneous oscillation and electrical stimulation.
\end{abstract}

Keywords - neuronal activation, brain metabolism, energy substrates, system identification.

\section{INTRODUCTION}

The human body is often referred to as a perfect mechanism, for each cause there is an effect, each signal triggers an action. The brain is undoubtedly the most complex and amazing organ; it represents a dense network of interconnections. Our understanding of the brain is still extremely limited, and probably will be for years to come. The neurobiological research, however, has allowed us to learn much about the functionality of the brain and has opened new means for understanding the cytoarchitectonic structure of this complex organ.

Understanding the biochemical and biophysical mechanisms, responsible for the functions of the human brain, is one of the most complex frontiers of science. The brain operates as the central control system for movement, sleep, hunger, and thirst. It controls nearly every vital activity necessary for survival. As there are no energy reserves in the brain, a constant supply of the main metabolic substrates, oxygen and glucose, is vital, and any interruption of the blood supply rapidly produces profound and irreversible changes of the brain function. A close interrelationship between metabolism and blood flow in the brain was first postulated by Roy and Sherrington in 1890 [1]. In recent years, this idea has been extended and verified. Global measurements of whole brain metabolism and blood flow have already demonstrated a direct relationship between functional activity, metabolism, and blood flow.

The more recent use of methods that allow determination of blood flow and metabolism in defined regions of the brain has revealed notable differences in blood flow between regions. This heterogeneous blood flow, as a result of varying local functions and metabolic levels, exists not only during resting conditions but also during brain activation, e.g., during the performance of mental tasks. The interpretation of the correlation between metabolism, blood flow and functional activity has led in recent years to a number of different hypotheses. First, the local metabolic rate for oxygen and glucose may depend on the degree of functional activity. Second, local blood flow may depend on the local metabolic rates for oxygen and glucose. Third, local blood flow may in fact depend on local functional activity which may be reflected in the EEG (electroencephalography) signal.

This paper will focus on prediction of rat brain activity (through the EEG signal from the rat barrel cortex), based on the simultaneous measurement of oxygen and glucose. The relationship between these signals will be elucidated in the following section where some compartmental models, based on metabolic mechanisms that occur during neuronal activation in anesthetized rats, are also discussed. 
Subsequently, a novel nonlinear mathematical model, representing the relationship between glucose, oxygen and EEG, is proposed and evaluated.

\section{CEREBRAL ACTIVITY AND BRAIN METABOLISM}

The application of various techniques, such as microdialysis, hydrogen clearance monitoring and electrode implantation, has enabled us to monitor the time course of metabolic changes during neuronal activation in animal experiments. Such experiments have shown that, after an electrical or mechanical stimulation, there is a very rapid increase in regional Cerebral Blood Flow (rCBF) and a delayed and more prolonged rise of glucose levels in the extracellular fluid.

Increased neuronal activity appears to evoke a triphasic response in the extracellular glucose concentration: an initial rapid fall, followed by an equally swift overshoot above the baseline and a subsequent return to baseline [2]. Furthermore studies using inhalation of radio-labelled oxygen have shown an uncoupling of regional cerebral glucose metabolism ( $\mathrm{rCM}_{\text {gluc }}$ ) and regional cerebral oxygen metabolism $\left(\mathrm{rCM}_{\mathrm{O} 2}\right)$ during brief increases in neuronal activity [3]. This uncoupling implies that glucose in the extracellular fluid is not directly derived from the blood flow and supports the hypothesis of metabolic compartmentation with glial cells playing an important role in the supply of metabolic substrates for neuronal metabolism [4].

Preliminary experiments that provide continuous, simultaneous measurement of $\mathrm{fCBF}$, oxygen and glucose present an important opportunity to elucidate the issue of energy supply and utilization. Lowry and Fillenz $[5,6]$ have previously measured $\mathrm{rCBF}$, tissue oxygen and extracellular glucose in the striatum of freely moving rats during neuronal activation and show evidence for uncoupling of oxygen and glucose utilization during neuronal activation in rat striatum. Rats were subjected to physiological stimulation with tail pinch. Attaching a paper clip on the tip of a rat's tail for 5 min produces a well characterized behaviour pattern which consists of gnawing, licking, eating and general increase in the level of motor activity.

This physiological stimulation increases neuronal activation in the striatum and a concurrent increase in rCBF was also noted. A close correspondence between the magnitude and time course of the increase in rCBF and oxygen, measured as a percentage of the basal values, also suggests that changes in oxygen are a reflection of the changes in rCBF; there also appears to be an increase in oxygen supply with no change in oxygen utilization. Since the oxygen current did not decrease below basal level at anytime, there appears to be no increased oxygen utilization either before or after the increase in $\mathrm{rCBF}$
In a separate group of rats changes in extracellular glucose were measured with a glucose oxidase electrode implanted in the right striatum. The glucose oxidase electrode provides a direct and continuous measure of extracellular glucose, which is the balance between supply and utilization. A 5\% decrease in glucose during the tail pinch experiment, represents an increase in utilization, since it occurs during the period of increased rCBF, and hence presumably not a fall in glucose supply. Furthermore, the absence of an increase in oxygen utilization suggests neuronal aerobic glycolysis. During this period there is an increase in extracellular lactate, which partly overlaps the decrease in glucose; the dependence of this increase in lactate on glutamate uptake suggests that it is derived from astrocytes. At the end of the tail pinch there was a slow rise in extracellular glucose which took $30 \mathrm{~min}$ to return to baseline level and occasionally glucose levels fell below basal level. The fact that the rise in glucose occurs when rCBF has returned to basal levels means that the rise in glucose cannot be derived directly from the bloodstream but must come from some other compartment. Evidence from a number of sources suggests that astrocytes constitute this compartment, as proposed by Magistretti in 1996. Figure 1 shows the neurovascular unit, the site of metabolic and hemodynamic changes.

From the studies, examined so far, emerge several considerations. Firstly, when neurons become active, they start to utilize the glucose and oxygen, and the increase of blood flow normally indicates the supply of the two substrates. Whereas when CBF returns back to normal, it shows that there are some compensatory mechanisms. Furthermore when the activation of cells is stopped, they became inactive, which usually signify that $\mathrm{CBF}$ is returning back to normal.

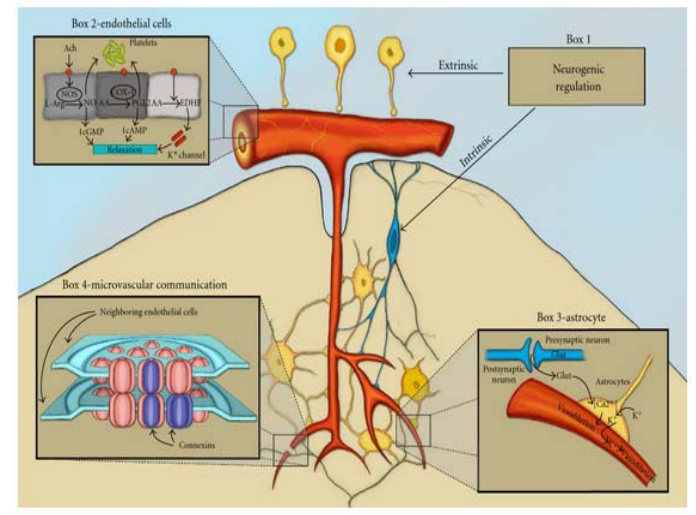

Figure 1: Comprehensive diagram demonstrating the multiple mechanisms of cerebrovascular control [7].

Regarding the level of oxygen in the tissue, studies in rats have shown a very close coupling with CBF. As shown in the study of the tail pinch $[5,6]$, after 5 minutes of stimulation, the CBF returns to baseline 


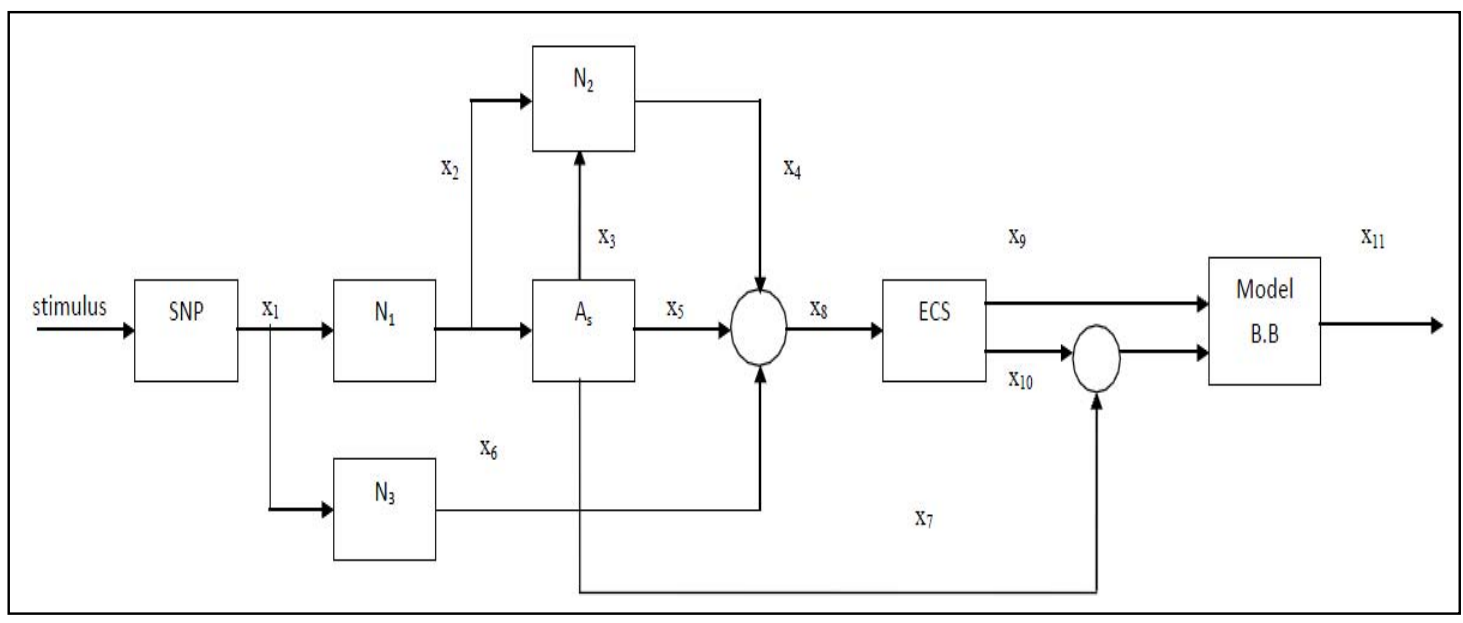

Figure 2: Anatomy-physiological model: stimulus is the neuronal activation; N1 and N2 are the family of neurons activate from parallel fibers; N3 includes neurons activated from climbing fibers; the ECS is the extracellular space, while the flows considered are the following: $\mathrm{x} 1$ is neuronal signal from afferent fiber, $\mathrm{x} 2$ is glutamate, $\mathrm{x} 3$ is arginina, $\mathrm{x} 4$ is $\mathrm{NO}, \mathrm{x} 5$ is $\mathrm{K}^{+}$, $\mathrm{x} 6$ is adenosine, $\mathrm{x} 7$ is glucose from astrocyte, $\mathrm{x} 8$ is $\mathrm{rCBF}, \mathrm{x} 9$ is oxygen in the extracellular space, $\mathrm{x} 10$ is glucose in the extracellular space and $\mathrm{x} 11$ is EEG signal.

and the oxygen also moves in the same direction. The glucose does not follow the same pattern, it does not seem to be coupled to the blood flow, in fact when the blood flow is back down to normal and the oxygen is back down to normal there is a sustained increase in the glucose which means that glucose actually comes from the astrocytes and not from the bloodstream. It is possible that there are in fact two sources of glucose - some glucose coming from the blood flow, and some glucose derived from the astrocytes.

There are probably two mechanism of removal of glucose. One mechanism would be to remove it by the storage compartment (for example in the astrocytes this is the glycogen), thus glucose is stored and in fact it can be broken down when the body requires it. The second mechanism is to remove glucose via the bloodstream: it comes from the blood, some is taken off by the astrocytes and some remains in the blood and is used by the neurons when they become active. Any redundant glucose, unused by the cells during activation is washed out by the bloodstream. In a similar manner the compartmental model suggests that lactate is exported from the astrocyte and taken up by the neurons. This is the most controversial part of the compartment model of the metabolism. Some researchers believe that lactate is not an energy substrate and it is not actually taken up by the neurons, it is just removed by the brain, because it is eliminated as a byproduct. The compartmental model assumes lactate is an energy substrate and that it is consumed by the neurons, while the classical model assumes that lactate is a metabolic byproduct. We need to remember that the lactate is produced by the brain and that the model of Magistretti suggests that in situations where there is little glucose or when cannot compensate for the energy demand quickly, glucose utilization is so high that the blood flow cannot compensate for the energy demand quickly, the lactate is used as an energy substrate. Today many believe that the role of lactate is a combination of the two models. Just like glucose coming from the bloodstream can go either into the extracellular space and be available to the neurons or it can go in the astrocytes where it is stored as glycogen and broken down later, the same possibility exist for lactate.

When lactate is produced it can either be removed as a byproduct by the bloodstream or it potentially could be taken up by the neurons, if they need it. The model that we proposed on the basis of the metabolic mechanisms in response to neuronal activation [8] is shown in the Figure 2.

\section{METHODS}

This present study focuses on the EEG, glucose and oxygen signals in the brain and develops a model capable to predict the EEG signal.

\section{a) Experimental set-up}

Two different experiments were carried out on anesthetized rats, one in the absence of stimulation and the other involving electrical stimulation of a whisker. The whisker barrel sensory pathway is an ideal network for this approach because it has topographical and columnar spatial organization, and well-defined vasculature, such that somatic stimulation produces discrete and reproducible responses within each cortical layer (see Figure 3).

The objective of both experiments is the same: to investigate the oxygen, glucose and EEG variations in the brain cortex. Both experiments used adult male Sprague-Dawley rats (280-350 g at the time of surgery). The rats were anesthetized with $1.5 \mathrm{~mL}$ of a solution of $25 \%$ urethane $(2 \mathrm{~g} / \mathrm{kg})$. The procedure involved an incision in the scalp and a resection of the periosteum. Five holes were made in the skull and the dura mater below was punctured with a hypodermic needle, then the working, reference and 
ground electrodes, for each signal were implanted. Implanting of amperometric micro-electrodes in a specific region of the brain, and applying a suitable potential, allows us to record the faradic current and monitor changes in the concentration of a variety of substances in the ECF for long periods $[9,10]$. Thus we can perform investigations on the functions and roles of specific neurochemical substances.

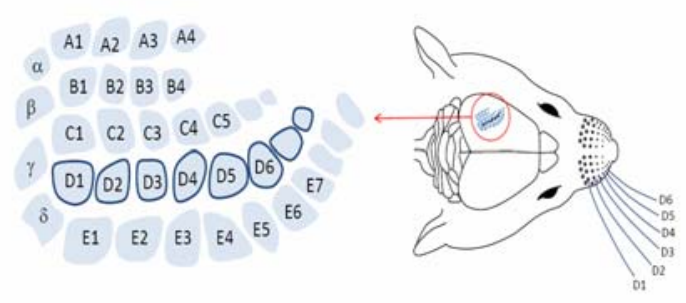

Figure 3: Reconstruction of recording sites in rat whisker barrel cortex.

\section{b) Preliminary data analysis}

After the oxygen, glucose and EEG data was recorded, it was resampled at $200 \mathrm{~Hz}$. Figure 4 shows a plot of the three signals recorded during the experiment.
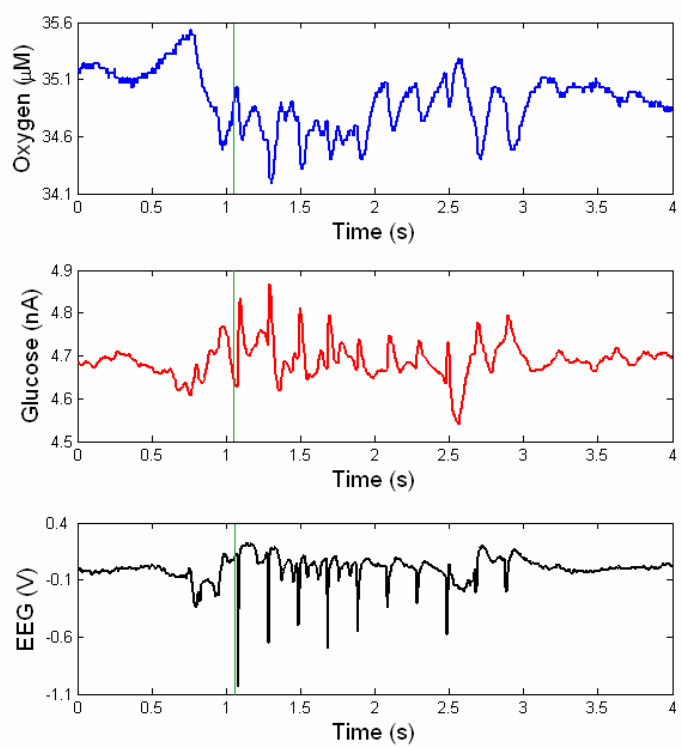

Figure 4: Evoketed response in the whisker barrel cortex, after a stimulation of $5 \mathrm{~Hz}$ (at Time $=1.1 \mathrm{~s}$ ), duration $2 \mathrm{sec}$, amplitude 1.2mA: Oxygen, Glucose and EEG signals are presented.

The first step was to analyze the correlation between the signals to try to find correspondence with what has been highlighted previously in the literature. As part of the analysis we need to assess the degree of linear dependency between the variables involved in the process [11]. All signals of interest (EEG, glucose and oxygen) showed correlation with various delays to one another. The coefficient of correlation measures the strength of the linear relationship between two signals; however many physiological systems exhibit nonlinear behaviour (e.g. variation in cerebral blood flow (CBF) during pacing, etc.), thus, in order to identify possible nonlinear relationships between our signals, we need to use an alternative measure suitable for nonlinear systems.

The mutual information is a statistical non-linear measure which is generally regarded as a measure of dependency between two random variables. If the two variables are independent, the mutual information between them is zero, if the two variables are strongly dependent the mutual information between them is large. The mutual information can be used to assess dependency in non-linear relationships. We can use this information to evaluate the possibility of selecting a set of optimal input variables for the system identification process [12]. Strong relationships were again found between the EEG, oxygen and glucose signals.

\section{c) Model structure specification}

Based on considerations drawn from the literature, and on a section of the model presented in Figure 2, we have proposed a new anatomy-physiological model that is useful for the identification of inputoutput models, aimed at resolving the problem of prediction of neuronal activation by changes of principal substrates involved in neuronal metabolism. In essence, the proposed model predicts the EEG signal, using the glucose and oxygen signals as measured inputs (see signals $\mathrm{X}_{9}, \mathrm{X}_{10}$ and $\mathrm{X}_{11}$ in the model in Figure 2).

Initially, a linear "black box" model with an ARMAX (Auto Regressive Moving Average with eXogenous input) [12] structure was considered. The response was assessed and it was deemed necessary to include some non-linear model components to account for certain features in the data.

A number of different nonlinear model structures were investigated. One structure allowed us to capture very well most features in the EEG data and is presented in Section IV.

\section{d) Model Parameterisation}

The model was parameterised using some "training data" - first $2 / 3^{\text {rds }}$ of the data set, while the latter $1 / 3^{\text {rd }}$ of the data samples were used for model validation.

The Nelder and Mead simplex method [13] was used to optimise the model parameters and the performance function used to obtain the best fit was the mean squared error (MSE).

\section{a) Model Structure}

\section{RESULTS}

The model developed in this study, predicts the EEG signal, using glucose and oxygen as inputs. The model is shown in Figure 5. The relationships between glucose and EEG and oxygen and EEG are captured by a set of first order transfer functions. 


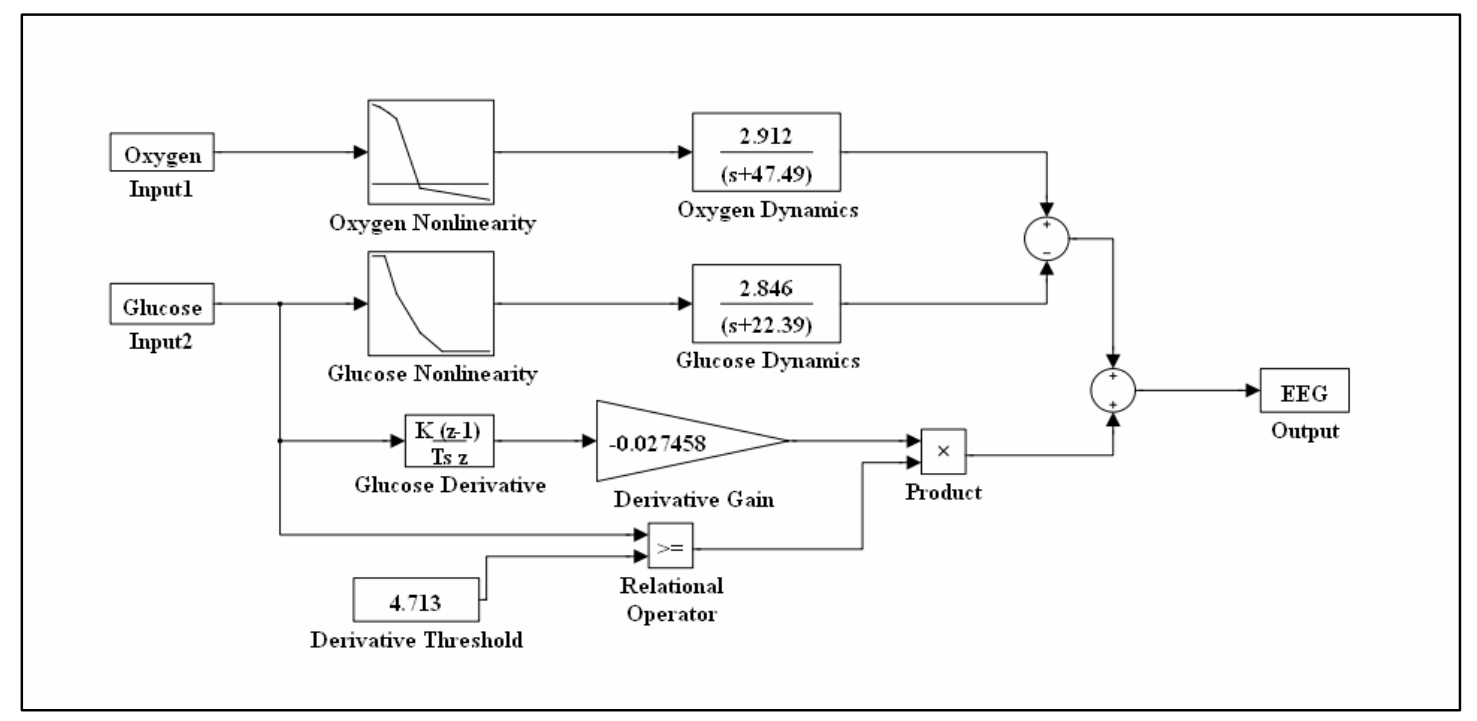

Figure 5 Nonlinear Model to predict EEG using glucose and oxygen as inputs

Each transfer function is preceded by a static nonlinearity (activation function). In addition, the inclusion of a differencing function (with a threshold and gain) on the glucose signal, in parallel with the two paths described above, allowed us to predict the negative peaks of the EEG signal.

\section{b) Model Fit}

The experimental work carried out in this study showed that a linear model for the prediction of an EEG signal based on the measurements of oxygen and glucose, and using an ARMAX structure, was able to reproduce some of the features of the EEG signal, but not the large peaks in data (Fig. 6). This is not too surprising, given that the model output (EEG) was constructed based on the more slowly varying input signals of oxygen and glucose.

Using the nonlinear model shown in Figure 5, we have succeeded in capturing most of the dynamics of the physiologic system analyzed here, as is shown in Figure 6.

Table 1 gives the final mean squared errors for the best-fit ARMAX model and the non-linear model shown in Figure 5.

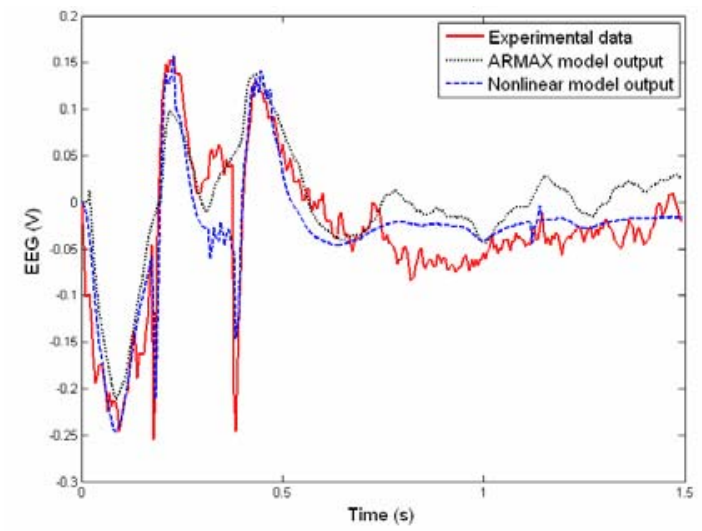

Figure 6: Model with static non-linear function for oxygen and glucose, considering the stimulation.
The two static nonlinear functions are in addition shown in Figure 7. They both manifest an inverse exponential-shaped response and represent activation levels for the effects of oxygen and glucose on the EEG signal. Such static nonlinear functions are commonly found in physiological systems [14] and are due to the fact that different concentration levels of one substance result in different corresponding levels of release/uptake of another substance (or in this case they result in different voltage levels).

\begin{tabular}{ll}
\hline Model Structure & MSE[V $\left.^{2}\right]$ \\
\hline ARMAX & $2.1 * 10^{-3}$ \\
Non-linear model & $1.28 * 10^{-3}$ \\
\hline
\end{tabular}

Table 1 Mean squared errors for the linear ARMAX model and the non-linear model shown in Figure 5.

\section{CONCLUSIONS}

The objective of this work was to predict the neural response (EEG) to whisker stimulation in the rat, using only the measured signals of glucose and oxygen in the brain as inputs. Such predictions can be particularly useful for the prevention, stabilization, or treatment of pathologies such as Alzheimer's disease, migraine headache, and ischemic stroke, in which signaling between neurons and brain vessels is threatened because of dysfunctions that affect the neuronal, astroglial, and/or vascular components of the neurovascular unit. To this end research is making great progress for developing biosensors based on telemetry systems that allow in real time the detection of levels of some energy substrates.

What emerges from this work is that with a model consisting of two simple first order transfer functions, which can capture the dynamics of the physiological system, two static non-linearities, and a differencing function on the glucose path, we can 
provide a satisfactory prediction of the EEG signal based on measurements of glucose and oxygen.
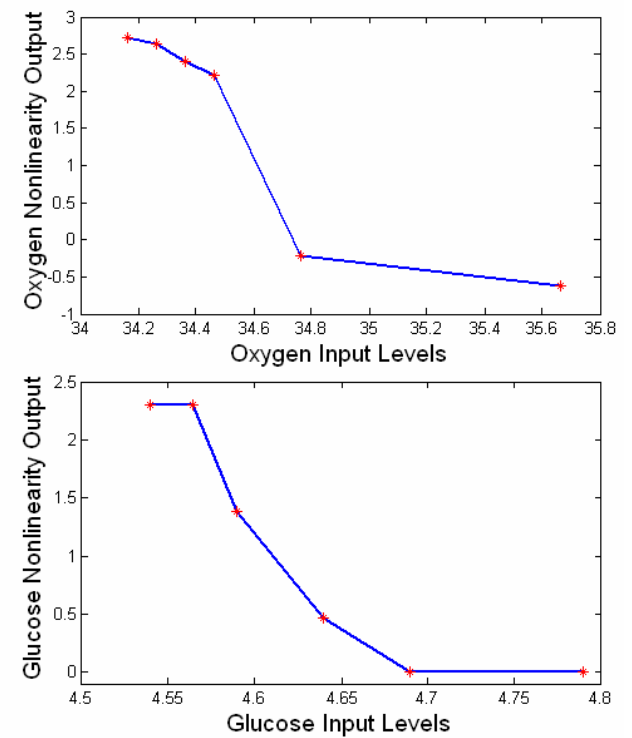

Figure 7: Static non-linearities for the glucose and oxygen signals

Despite the good model fit and credible static nonlinearities, we need to note that oxygen and glucose are not unique substrates for the regulation of metabolism in the brain. There are other intermediate variables, such as nitric oxide, noradrenaline and lactate, that play an important role in brain metabolism and could be considered as possible explanatory variables also.

The modeling approach and model structure employed in this study were subsequently applied in another study, which modelled EEG, glucose and oxygen data with spontaneous oscillations (i.e. there was no electrical whisker stimulation). The model was not always able to predict the spontaneous EEG signal to the same level, as it did in this present study; however this could be due to the more complex EEG signal in the case of electrical whisker stimulation. The EEG signal is generally a predictable signal, since it only indicates that the cells are active (it is an electrical activation), consequently, we can assume that it is the neuronal chemistry that is not very predictable, as even though we can see how the two substrates (oxygen and glucose) are changing, we cannot be certain what the likely causes of these variations are (the possibilities include NO, rCBF, etc.). Thus, it is necessary to continue research towards even more complete models that take into account a more comprehensive set of interacting physiological processes in the body, to avoid falling into the trap of creating selective models suited to specific species/situations only. In addition, there is a very strong possibility that the glucose-oxygen-EEG signals in fact operate in a closed loop system, so in future work this will need to be investigated to allow for a more truthful model of the system.
Finally it is important that potential future work would be directed towards greater interaction between Bioengineering and Biochemistry to permit us to converge on a mathematical model, which is more comparable with the data from multiple experimental protocols and whose parameters can be more easily interpreted and related to the underlying physiological system.

\section{REFERENCES}

[1] C. S. Roy and C. S. Sherrington, "On the Regulation of the Blood-supply of the Brain”. J Physiol. 11(1-2): [85-108, 158-7-158-17], 1890

[2] I.A. Silver and M. EreciAska, "Extracellular glucose concentration in mammalian brain: Continuous monitoring of changes during increased neuronal activity and upon limitation in oxygen supply in normo, hypo-, and hyperglycemic animals”, The Journal of Neuroscience, 14, 5068-5076, 1994

[3] L. Sokoloff, "The brain as a chemical machine”, Progress in Brain Research, l 94, 19-33, 1992.

[4] M. Tsacopoulos and P. Magistretti, "Metabolic coupling between glia and neurons", Journal of Neuroscience, 16, 877-885, 1996.

[5] J . P. Lowry and M. Fillenz, "Evidence for uncoupling of oxygen and glucose utilization during neuronal activation in rat striatum, Journal of Physiology, 498 (2), 497-501, 1997.

[6] J . P. Lowry and M. Fillenz, "The relation between local cerebral blood flow and extracellular glucose concentration in rat striatum”, Experiment Physiology, 83, 233-238, 1998.

[7] E.C. Peterson, Z.Wang and G. Britz, "Regulation of Cerebral Blood Flow", International Journal of Vascular Medicine, vol 2011, pp 8.

[8] M. Fillenz, J.P Lowry, M.G Boutelle, and E.A. Fray, "The role of astrocytes and noradrenaline in neuronal glucose metabolism”, Acta Physiologica Scandinavica, 284, 167-275, 1999.

[9] J. Li, D.S. Bravo, A.L Upton, G.G. Gilmour, P.L. Lowry, D.M. Bannerman, and S.M. McHugh, "Close temporal coupling of neuronal activity and tissue oxygen responses in rodent whisker barrel cortex”. European Journal of Neuroscience, 34, 19831996, 2011

[10] Lowry J.P, "Monitoring Real-time Metabolite Trafficking in the Brain using Microelectrochemical Biosensors”.

[11] A. V. Lefebvre, I. Zheng, I.M. Devonshire, and C.J Martin, "Investigation the coupling between stimulation and neural activity: a dynamic modeling approach”, In Conference of the IEEE EMBS, 2007.

[12] L. Ljung, "System Identification-Theory For the User”, $2^{\text {nd }}$ ed, PTR Prentice Hall, Upper Saddle River, N.J., 1999 .

[13] J. Nelder and R. Mead. “A simplex method for function minimization". The Computer Journal, 7 (4):308-313, 1965.

[14] V. Mangourova, J. V. Ringwood, S.-J. Guild and S. Malpas. "Nonlinear modelling of renal vasoaction”. Biomedical Signal Processing and Control, 2,258-266, 2007. 